\title{
Outcomes of a multimodal cognitive and physical rehabilitation program for persons with mild dementia and their caregivers: a goal-oriented
} approach

\author{
This article was published in the following Dove Press journal: \\ Clinical Interventions in Aging \\ 22 October 2015 \\ Number of times this article has been viewed
}

\section{Justin Chew \\ Mei-Sian Chong \\ Yoke-Leng Fong \\ Laura Tay}

Department of Geriatric Medicine, Institute of Geriatrics and Active Ageing, Tan Tock Seng Hospital, Singapore, Singapore
Correspondence: Justin Chew Department of Geriatric Medicine, Tan Tock Seng Hospital, Annex 2, Level 3, II Jalan Tan Tock Seng, Singapore 308433, Singapore

Tel +6563596474

Fax +6563596274

Email justin.chew@mohh.com.sg
Background: Nonpharmacological interventions such as exercise and cognitive rehabilitation programs have shown promise in reducing the impact of dementia on the individual and the caregiver. In this study, we examine the effect of a multimodal cognitive and physical rehabilitation program for persons with mild dementia and their caregivers using conventional measures of cognition, behavior, quality of life (QoL), and caregiver burden together with goal attainment scaling (GAS), an individualized outcome measure.

Methods: Goals were set at baseline, and GAS score was calculated at the end of the program. Participants were also assessed with the Chinese Mini-Mental State Examination, functional and behavioral scales (Barthel Index), Instrumental Activities of Daily Living, Neuropsychiatric Inventory Questionnaire, QoL, and caregiver burden using EuroQol-five dimension questionnaire and Zarit Burden Interview (ZBI). Differences in median scores postintervention were obtained. Further analysis of caregiver burden was undertaken utilizing the multidimensional classification of burden on the ZBI.

Results: Thirty-four (61.8\%) patients were assessed to have met their goals (GAS score $\geq 50$ ). Mean (standard deviation) GAS score was 48.6 (6.5). Cognition goals were set in only $20.6 \%$, followed by goals to improve engagement and socialization; reduce caregiver stress; and improve physical function, behavior, and mood. Median scores in the cognitive, functional, and QoL measures did not differ significantly pre- and postintervention. The intervention had a positive impact on role strain, a unique dimension of caregiver burden.

Conclusion: This study provides evidence that a multimodal approach combining physical exercise and cognitive rehabilitation improves goal attainment and caregiver burden in individuals and caregivers of persons with mild dementia.

Keywords: dementia, rehabilitation, goal attainment scaling

\section{Introduction}

As the number of persons living with dementia increases, there is a need to develop effective interventions for Alzheimer's disease (AD). The fact that no new AD drug has been approved since $2004^{1}$ underscores the importance of interventions aimed at improving the quality of life (QoL) of people living with dementia and the support given to their caregivers. ${ }^{2}$ To this end, various nonpharmacological approaches have been employed, such as physical exercise and cognition-focused interventions. A growing body of literature suggests that exercise has a positive effect on cognition. ${ }^{3}$ Cognitive rehabilitation is another modality of treatment that focuses on finding strategies to maintain or cope with memory impairment, with the aim of decreasing 
functional disability and maximizing social participation and engagement in activities of daily living (ADL). ${ }^{4}$

The impetus for this study is twofold. While there are studies evaluating the effects of individual interventions, less evidence exists on multimodal nonpharmacological approaches. ${ }^{5}$ In particular, the impact of exercise programs and cognitive interventions on caregiver burden is less well defined. Second, in dementia trials, there is a need to utilize instruments that are able to capture clinically important treatment effects. Drug trials have traditionally pursued cognitive and functional abilitybased end points, in part owing to regulatory fiat as the US Food and Drug Administration still requires the demonstration of efficacy in cognitive and functional domains. ${ }^{6} \mathrm{~A}$ four-point change in the Alzheimer's Disease Assessment Scale-Cognitive Subscale $^{7}$ is held to be a criterion for clinical significance, but the justification for this threshold is debatable. ${ }^{8}$

Isolated cognitive instruments or global measures that approximate an overall level of impairment may not adequately reflect treatment effects that are important to an individual and caregiver. A different approach may thus be required to shift the focus to clinically relevant outcomes. Goal attainment scaling (GAS) is an individualized approach to measure clinical outcomes that was first introduced by Kiresuk et $\mathrm{al}^{9}$ for evaluating community mental health programs. It is a process of defining individual treatment goals at the outset and monitoring for goal attainment. Goals are comparable as results can be summarized using a formula. GAS provides the means for comparing individuals at various stages and presentations of disease, and is adaptable across different populations and interventions, as shown in trials of antidementia drugs ${ }^{10,11}$ and cognitive rehabilitation. ${ }^{12}$

Our primary aim is to evaluate the efficacy of a multidisciplinary cognitive and physical rehabilitation program for elderly persons with mild dementia using conventional measures of cognition, functional performance, behavior, QoL, and caregiver burden, together with an individualized, goaloriented approach to measuring outcomes using GAS. ${ }^{9}$ The secondary objective is to study the effect of such an intervention on caregiver burden, highlighting the specific dimensions of burden relevant to caregivers of persons with dementia.

\section{Methods}

\section{Study participants}

This is a prospective observational cohort study involving community-dwelling elderly persons with mild dementia attending the MINDVital rehabilitation program in the outpatient Geriatric Clinic, Tan Tock Seng Hospital, Singapore, between June 2012 and March 2014. Participants were excluded if they did not have a caregiver. Enrollment in the program was based on referral by the participant's attending geriatrician, with participation being voluntary. Mild dementia was defined according to the Diagnostic and Statistical Manual of Mental Disorders, Third Edition, Revised criteria. Dementia etiology was assigned using the National Institute of Neurological and Communicative Disorders and Stroke and the Alzheimer's Disease and Related Disorders Association criteria for AD, ${ }^{13}$ and the International Workshop of the National Institute of Neurological Disorders and Stroke-Association Internationale pour la Recherche et I'Enseignement en Neurosciences ${ }^{14}$ criteria for vascular dementia (VaD). Participants with non-AD or non$\mathrm{VaD}$ diagnoses or with mixed etiology were classified under the category "others". Ethics approval was obtained from the National Healthcare Group Domain Specific Review Board for a retrospective data review.

\section{MINDVital}

MINDVital is a multidisciplinary rehabilitation program comprising once-weekly group therapy sessions, each session lasting 3 hours over 8 consecutive weeks. To ensure that each participant receives individualized attention, group sizes were limited to ten participants. A trained nurse coordinator on-site provides overall supervision, and sessions are facilitated by a multidisciplinary team of physiotherapists, occupational therapists, and psychologists.

All participants and their caregivers attend a brief interview at the beginning of the program to define their individual treatment goals. Each weekly session includes the following activities, with scheduled breaks between: 1) 45-minute multicomponent physical exercise program of light aerobic exercises, range of motion and resistance exercises, as well as balance training; 2) 1-hour cognitive stimulation and rehabilitation with social and mental activities such as reminiscence therapy; and 3) 30-minute tailored individualized activities delivering person-centered care.

Regular feedback on progress is provided to the participant and caregiver in the form of a progress card during the program, with advice to continue the learned activities at home between therapy sessions. At the end of the program, goal attainment is reviewed and scored.

\section{Measures}

\section{Assessment measures}

Participants were assessed pre- and postintervention with the cognitive scale Chinese Mini-Mental Status Examination, ${ }^{15}$ functional and behavioral scales using the Barthel Index, ${ }^{16}$ Instrumental Activities of Daily Living, ${ }^{17}$ Neuropsychiatric 
Inventory-Questionnaire (NPI-Q), ${ }^{18}$ QoL and caregiver burden using EuroQoL-five dimension questionnaire ${ }^{19}$ and Zarit Burden Interview (ZBI). ${ }^{20}$

To further explore the different domains of caregiver burden that may impact on QoL, we used four subscales of the ZBI as identified in our previous study: ${ }^{21}$ factor 1 -demands of care and societal impact on caregiver (role strain); factor 2 - confidence or control over the situation (role strain); factor 3 - psychological impact on caregiver (personal strain); and factor 4-worry about caregiving performance.

\section{GAS}

GAS is a clinimetric measure that evaluates treatment effects relevant to an individual. ${ }^{9}$ It is a process of defining individual treatment goals at the outset and monitoring for goal attainment. Goals are comparable across individuals and populations as the results can be summarized using a formula.
The process of goal setting begins at the initial interview with the participant and their caregiver, which first involves the identification of issues that will be the focus of treatment. The identified problems are then translated into goals, without restriction on the types of goals that can be set. The next step is to specify the expected level of outcome of the intervention for each goal, where possible outcomes are much less than expected $(-2)$, somewhat less than expected $(-1)$, expected $(0)$, somewhat better than expected $(+1)$, or much better than expected $(+2)$. The criteria for scoring at each level are determined in an observable way and described specifically. Participants are reviewed at the end of the intervention and scored on the basis of this continuum of possible outcomes. Follow-up assessment of goal attainment involves a summary GAS score, which is converted to a standardized T-score via the formula presented in Table 1. A GAS score of 50 is, by definition, an indication of overall goal attainment at

Table I Goal attainment scaling formula and sample of goal setting

$$
T=50+\frac{10 \sum w_{i} x_{i}}{\sqrt{(1-\rho) \sum w_{i}^{2}+\rho\left(\sum w_{i}\right)^{2}}}
$$

Where $x_{i}$ represents the attainment score for each goal (a value from -2 to +2 ), and $w_{i}$ represents the weight assigned to a particular goal. As goals in this study were unweighted, $w_{i}=1 . \rho$-value reflects the expected correlation of attainment scores, which is assumed to be 0.30 . In practice, the GAS T-score can be directly obtained from a summary score conversion table, ${ }^{\text {a }}$ doing away with the need for manual calculations

\begin{tabular}{|c|c|c|c|}
\hline Domain & Current & Goal & 8-week review \\
\hline \multicolumn{4}{|l|}{ Cognition (reality orientation with cues, eg, clocks, calendar) } \\
\hline \multicolumn{4}{|l|}{ Self-initiated reality orientation $>\mid$ time daily $(+2)$} \\
\hline \multicolumn{4}{|l|}{ Self-initiated reality orientation I time daily $(+I)$} \\
\hline Caregiver-initiated reality orientation $>\mid$ time daily $(0)$ & & $\checkmark$ & $\checkmark$ \\
\hline \multicolumn{4}{|l|}{ Caregiver-initiated reality orientation I time daily $(-1)$} \\
\hline Not oriented, no reality orientation $(-2)$ & $\checkmark$ & & \\
\hline \multicolumn{4}{|l|}{ Carer stress } \\
\hline \multicolumn{4}{|l|}{ No carer stress $(+2)$} \\
\hline Very occasional, $<I$ time a month $(+\mathrm{I})$ & & & $\checkmark$ \\
\hline Minimal stress $(0)$ & & $\checkmark$ & \\
\hline Moderate stress $(-1)$ & $\checkmark$ & & \\
\hline \multicolumn{4}{|c|}{ Extreme stress, not coping $(-2)$} \\
\hline \multicolumn{4}{|c|}{ Engagement in leisure activities (watercolor painting and Chinese calligraphy) } \\
\hline \multicolumn{4}{|c|}{ Leisure activities for 30 minutes daily $(+2)$} \\
\hline Leisure activities for 30 minutes $>3$ times a week $(+\mathrm{I})$ & & & $\checkmark$ \\
\hline Leisure activities for 15 minutes $>3$ times a week $(0)$ & & $\checkmark$ & \\
\hline Leisure activities for 15 minutes $<3$ times a week $(-1)$ & $\checkmark$ & & \\
\hline \multicolumn{4}{|l|}{ No initiation of activities $(-2)$} \\
\hline \multicolumn{4}{|l|}{ Sleep } \\
\hline \multicolumn{4}{|l|}{ Sleep 6-8 hours a night $(+2)$} \\
\hline \multicolumn{4}{|l|}{ Sleep 4-5 hours a night $(+I)$} \\
\hline Sleep $2-3$ hours a night $(0)$ & & $\checkmark$ & \\
\hline Sleep less than 2 hours a night $(-1)$ & $\checkmark$ & & $\checkmark$ \\
\hline No sleep $(-2)$ & & & \\
\hline
\end{tabular}

Note: ${ }^{a}$ Data from Kiresuk et al. ${ }^{9}$

Abbreviation: GAS, goal attainment scaling. 
the expected level. Scores greater than 50 indicate a betterthan-expected outcome, and scores less than 50 indicate a less-than-expected outcome.

Examples of GAS for cognition, caregiver stress, engagement in leisure activities, and sleep goals are shown in Table 1. For carer stress, if a goal was set at the level of "minimal stress", then a score of 0 was assigned, indicating the expected outcome. At the end of 8 weeks, if the goal was exceeded as described "very occasional, $<1$ time a month", then a score of +1 would be obtained, and +2 if the goal of "no carer stress" was achieved. Scores of -1 or -2 were given at described levels below the expected outcome.

\section{Statistical analysis}

Descriptive data are presented as mean \pm standard deviation (SD) or median (interquartile range, IQR) for quantitative variables, and as absolute and relative frequencies for categorical variables.

The Wilcoxon signed-rank test was used to compare cognitive and functional performance, neuropsychiatric symptoms, QoL, and caregiver burden including the fourfactor structure of the ZBI pre- and postintervention. Effect sizes were calculated as an index of responsiveness of the outcome measures used in this study. Cohen's $d$ was used to obtain effect size estimates for GAS scores, calculated by dividing change in scores by pooled SD (population standard deviation). ${ }^{22}$ For nonparametric data, effect size estimates $r$ were calculated with the formula $r=z / \sqrt{ } N$ using the $z$ score of the Wilcoxon test and $N$, the total number of observations. ${ }^{23}$

Correlation between GAS scores and other assessment measures was calculated using Spearman rank correlation coefficients. Statistical analyses were performed using IBM SPSS Statistics for Windows, Version 20.0 (IBM Corporation, Armonk, NY, USA; 2011). Statistical significance was taken to be $P<0.05$.

\section{Results}

This study included 55 participants, with a mean age of $79 \pm 6.3$ years. Forty-six percent of the participants were female. All the participants were diagnosed with mild dementia, and the etiology of dementia was AD in $65 \%, \mathrm{VaD}$ in $13 \%$, and mixed in $22 \%$ of the participants. Approximately $69 \%$ of the participants were on cognitive enhancers (cholinesterase inhibitors or memantine) for the duration of the intervention. The mean number of years of education was $9 \pm 5$. Table 2 shows the main characteristics of the study participants.
Table 2 Participant baseline characteristics $(n=55)$

\begin{tabular}{ll}
\hline Characteristic & Value \\
\hline Age, mean \pm SD & $79.2 \pm 6.3$ \\
Female, $n(\%)$ & $25(46)$ \\
Years of education, mean \pm SD & $9 \pm 5$ \\
Cognitive enhancers for dementia, $\mathrm{n}(\%)$ & $38(69)$ \\
Etiology of dementia, $\mathrm{n}(\%)$ & \\
Alzheimer's disease & $36(65)$ \\
Vascular dementia & $7(13)$ \\
Others & $12(22)$ \\
Median scores (interquartile range) & \\
CMMSE & $18(15-21)$ \\
Barthel Index & $100(95-100)$ \\
IADL & $1 \mathrm{II}(7-15)$ \\
EQ-5D & $5(5-5)$ \\
NPI-Q severity & $2(1-4)$ \\
NPI-Q caregiver distress & $\mathrm{I}(0-3)$ \\
ZBI & $16(9-29)$ \\
\hline
\end{tabular}

Abbreviations: SD, standard deviation; CMMSE, Chinese Mini-Mental State Examination; IADL, Instrumental Activities of Daily Living; EQ-5D, EuroQoL-five dimension questionnaire; NPI-Q, Neuropsychiatric Inventory Questionnaire; ZBI, Zarit Burden Interview.

The average number of goals set by the participants and their caregivers was 2.6. Cognition goals were most frequently identified, but comprised only $21 \%$ of all the goals set. The next most common goals identified were aimed at improving engagement and socialization, followed by caregiver stress, physical function, behavior, mood, and sleep. Unmet cognition goals were most frequently observed. Figure 1 shows the different goals set and proportion of goals attained.

Overall, the mean GAS score postintervention was 48.6 \pm 6.5 . Sixty-two percent of participants met or exceeded their goals at the end of the intervention, as defined by a GAS score of 50 and above. Ninety-six percent of goals to increase engagement and socialization and $94 \%$ of goals to improve mood were met, followed by $92 \%$ of goals to reduce caregiver stress, $81 \%$ for behavioral and functional goals, and $55 \%$ of cognition goals were attained at the end of the intervention, also shown in Figure 1.

As shown in Table 3, scores in the cognitive, function, and QoL did not differ significantly pre- and postintervention. Effect sizes for cognitive scores, functional performance, neuropsychiatric symptoms, QoL, and caregiver burden measures were marginal, whereas a large effect size (Cohen's $d=2.53$ ) was seen for GAS.

There was a trend for improvement in caregiver burden postintervention, with median ZBI score of 16 (IQR: 9-29) at baseline, and 14 (IQR: 6-26) at 8 weeks $(P=0.080)$. When caregiver burden was analyzed according to the distinct fourfactor structure of the ZBI, factors 1 (demands of care and 


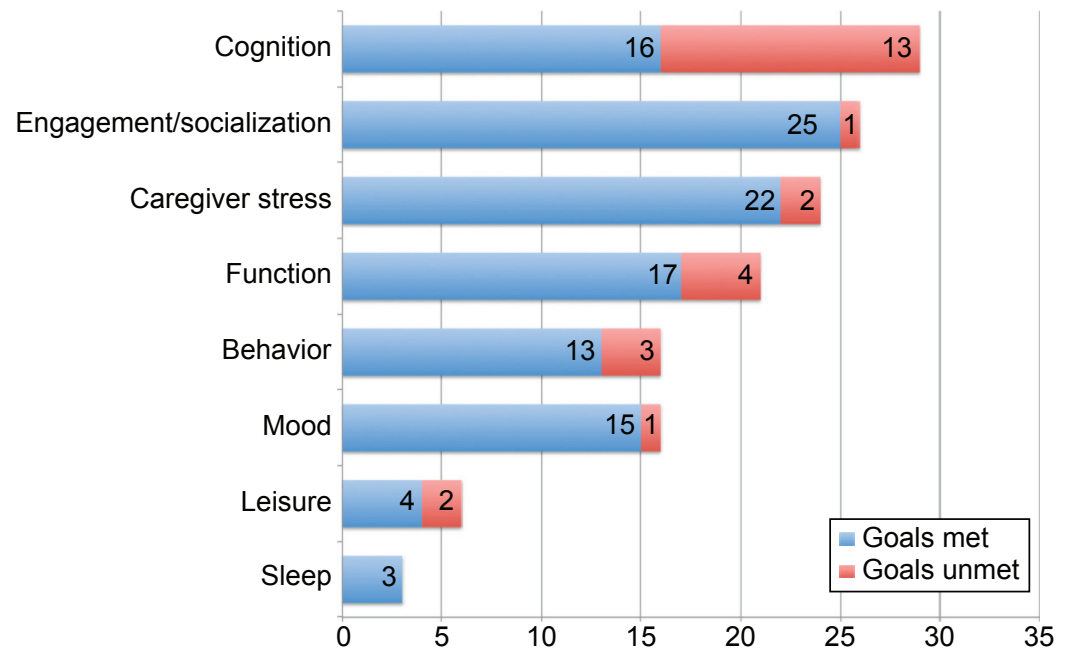

Figure I Types and number of goals met or unmet.

social impact) and 2 (confidence or control over the situation) revealed statistically significant improvement postintervention, as shown in Table 3.

GAS significantly correlated with improvements in caregiver burden on the ZBI and behavior severity on the NPI-Q as shown in Table 4, although the correlation was modest.

\section{Discussion}

Despite the stability of scores on standard scales measuring cognition, function, and behavior, over half of the persons with mild dementia in our cohort achieved or exceeded their goals following a multimodal cognitive and physical rehabilitation program. Goals to improve socialization, mood, and caregiver burden were most frequently attained. Contrary to the conventional emphasis placed on cognitive abilities in dementia trials, cognitive goals constituted only $21 \%$ of goals set. In particular, we observed that goals to reduce caregiver burden comprised a significant proportion of goals set. Nonpharmacological interventions have potential benefit in persons with dementia and their caregivers. Physical activity may reduce the risk of progression of ADL disability in patients with dementia, ${ }^{24}$ with exercise conferring benefits on behavioral and psychological symptoms of dementia, especially depressed mood, agitation, and wandering, and may also improve nighttime sleep. ${ }^{25}$ Cognitive interventions share the common underpinning of cognitive and neural plasticity in AD. ${ }^{26}$ Participants in MINDVital undergo cognitive stimulation and rehabilitation, an approach that also emphasizes a collaborative process between the caregiver and the person living with dementia, with intervention developed to address personally meaningful goals relevant to daily living. ${ }^{27}$

Combining nonpharmacological approaches in dementia rehabilitation has shown promise. In an AD rehabilitation program involving physical exercise and cognitive stimulation activities, a 4-year intervention group showed no decline

Table 3 Comparison of scores pre- and postintervention, with effect size estimates for each measure

\begin{tabular}{|c|c|c|c|c|}
\hline Measure & Preintervention & Postintervention & $P$-value & Effect size \\
\hline$\overline{\mathrm{GAS}^{\mathrm{a}}}$ & $36.8(1.0)$ & $48.6(6.5)$ & $<0.001$ & 2.53 \\
\hline $\mathrm{CMMSE}^{\mathrm{b}}$ & $18.0(|5.0-2| .0)$ & $19.0(\mid 5.8-21.3)$ & 0.14 & 0.14 \\
\hline Barthel Index & $100(95-100)$ & $100(95-100)$ & 0.60 & 0.05 \\
\hline IADL & $11(7-15)$ & $12(8-14)$ & 0.24 & 0.11 \\
\hline EQ-5D & $5(5-5)$ & $5(5-5)$ & - & - \\
\hline ZBI total & $16(9-29)$ & $14(6-26)$ & 0.080 & -0.17 \\
\hline Factor I (demands of care and social impact) & $8(4-16)$ & $7(2-7)$ & 0.046 & - \\
\hline Factor 2 (confidence or control over the situation) & $\mathrm{I}(\mathrm{I}-5)$ & $0(0-2)$ & 0.004 & - \\
\hline Factor 3 (psychological impact on caregiver) & $3(I-7)$ & $2.5(0-6.25)$ & 0.068 & - \\
\hline Factor 4 (worry about caregiving performance) & $3(2-4)$ & $3(2-4)$ & 0.899 & - \\
\hline NPI-Q severity & $2(I-4)$ & I $(0-3)$ & 0.24 & -0.11 \\
\hline
\end{tabular}

Notes: aMean ( \pm SD) for GAS scores. 'Median (IQR) for CMMSE, Barthel, IADL, EQ-5D, Zarit, and NPI-Q severity scores.

Abbreviations: GAS, goal attainment scaling; CMMSE, Chinese Mini-Mental State Examination; IADL, Instrumental Activities of Daily Living; EQ-5D, EuroQoL-five dimension questionnaire; NPI-Q, Neuropsychiatric Inventory Questionnaire; ZBI, Zarit Burden Interview; IQR, interquartile range; SD, standard deviation. 
Table 4 Spearman correlation coefficients for change scores and GAS scores

\begin{tabular}{lll}
\hline Change score & Correlation coefficient & $\boldsymbol{P}$-value \\
\hline Barthel Index & -0.155 & $0.28 \mathrm{I}$ \\
IADL & -0.089 & 0.540 \\
EQ-5D & -0.179 & 0.214 \\
NPI-Q severity score & $-0.46 \mathrm{I}$ & 0.001 \\
ZBI & -0.352 & 0.013 \\
\hline
\end{tabular}

Abbreviations: IADL, Instrumental Activities of Daily Living; EQ-5D, EuroQoLfive dimension questionnaire; NPI-Q, Neuropsychiatric Inventory Questionnaire; ZBI, Zarit Burden Interview; GAS, goal attainment scaling.

in several cognitive and language outcome measures. ${ }^{28}$ In another pilot 3-month program consisting of cognitive stimulation, physical activity, and socialization, the intervention group demonstrated stability of cognition and marked improvement in apathy, mood, and QoL compared to a control group. ${ }^{29}$ It is notable that while we had failed to demonstrate significant improvements in cognitive, behavioral, and functional scores, a majority of these individual set goals were actually met at the end of the rehabilitation program.

In our cohort, caregiver stress was identified during goal setting in a significant proportion of participants, despite the low median score on the ZBI that would correspond to the experience of no-to-mild burden. It is increasingly recognized that caregiver burden is a multidimensional concept that goes beyond the total score on the ZBI. ${ }^{30}$ Our intervention impacted two distinct dimensions of caregiver burden: 1) the demands of care or social impact on the caregiver and 2 ) the confidence or control over the caregiving situation. These two factors reflected the concept of role strain, broadly defined as the caregiver's felt difficulty in fulfilling the caregiver role. Predictors for role strain have previously been identified - mutuality, defined as the ability to find gratification and meaning in the relationship with the person living with dementia and the caregiving situation, and preparedness for caregiving, pertaining to one's perceived self-efficacy in managing the problems that come with dementia caregiving. ${ }^{31}$ As education and engagement were a key component of our intervention with encouragement to continue learned activities at home, this may have contributed to a greater degree of confidence in caregiving. We further hypothesize that the involvement of the caregiver through goal setting is itself a facilitative process, and may indirectly contribute to the reduction in role strain by reinforcing the benefits of mutuality through shared decision-making and goal setting.

The use of GAS in this study enabled an individualized approach to measuring outcomes and may have better reflected clinically relevant change. Measuring goal attainment in cognitive rehabilitation with GAS may be particularly appropriate, due to the personalized nature of cognitive rehabilitation goals. ${ }^{27,32}$ The psychometric properties of GAS have previously been described. ${ }^{9}$ GAS is a responsive outcome measure, as seen in the high effect size statistic consistent with previous studies. ${ }^{33}$ GAS also has good interrater reliability, ${ }^{34}$ and is feasible and acceptable to both individuals and caregivers. ${ }^{35}$ The intrinsic properties of GAS render evaluations of validity more complex as the individual domains identified would necessarily vary across diseases and populations. It is hence suggested that content validity be assessed on a case-by-case basis. ${ }^{36}$ Construct validity is usually evaluated via correlations with standard outcome measures. Similar to previous studies, ${ }^{35,37}$ we found that GAS correlations with standardized tests in our cohort were expectedly variable. As GAS utilizes individual goals, it may be less relevant to compare GAS with a construct using the same items across subjects. Nonetheless, in our study, GAS correlated significantly with improvements in caregiver burden on the ZBI and behavior severity on the NPI-Q, which highlights the potential utility of GAS in these domains.

\section{Limitations}

Limitations of this study include its lack of comparator group, small sample size, short duration of intervention, and limited generalizability beyond mild dementia. The degree to which different interventions contribute to goal attainment and caregiver burden is also not apparent. A potential concern of using GAS is appropriate scaling, although this problem is not unique to our study. Care has to be taken to ensure that goals set are neither too easily attainable nor too difficult to achieve. The higher proportion of unmet cognition goals in our study possibly reflected goals that may have been too difficult to attain. In our cohort, unattained cognition goals were nonspecific, nondescriptive goals targeted at improvements in cognitive scores, which highlighted the importance of appropriate, guided goal setting. On the other hand, this process allowed us to glean new insights on how the illness and treatment is perceived at an individual level, and hence better manage the expectations and concerns of the patient and caregiver.

\section{Conclusion}

In conclusion, our data provide preliminary evidence of the efficacy of a multimodal exercise and cognitive rehabilitation program for persons with mild dementia, particularly on goal attainment and caregiver burden. A goal-oriented approach may be more relevant in dementia trials, given the clinical heterogeneity of the AD syndrome, where deficits may be 
subtle and may not be reflected in standard scales. Education level, cultural perceptions, and the social environment may also influence treatment outcomes. Thus, a goal-oriented approach yielded more clinically relevant information on treatment effects and outcomes important to the individual and caregiver, beyond producing statistically significant improvements in routine neuropsychological tests and global functional outcomes. Further studies over a longer duration and a larger sample could be undertaken to determine the effects of multimodal interventions on reducing cognitive decline and the impact of dementia on the individual and the caregiver.

\section{Acknowledgments}

We would like to thank all the participants and caregivers of the MINDVital program. We also gratefully acknowledge all the members of the MINDVital team, including dementia care nurse clinicians Philomena Anthony and Han Huey Charn, Agnes Teo and her team of physiotherapists, Pamela Chew and June Lim, Sabrina Ow and her team of occupational therapists, and Sharon Wee for her editorial assistance.

\section{Disclosure}

Dr Tay was supported by the Tan Tock Seng Hospital Pitchfor-Fund grant in 2012 for a pilot study evaluating the impact of MINDVital on physical performance in early Alzheimer's disease. The authors report no other conflicts of interest in this work.

\section{References}

1. Cummings JL, Morstorf T, Zhong K. Alzheimer's disease drugdevelopment pipeline: few candidates, frequent failures. Alzheimers Res Ther. 2014;6(4):37.

2. World Health Organization and Alzheimer's Disease International. Dementia: A Public Health Priority. Geneva, Switzerland: World Health Organization. Available from: http://www.who.int/mental_health/ publications/dementia_report_2012/en/. Accessed July 1, 2015.

3. Heyn P, Abreu BC, Ottenbacher KJ. The effects of exercise training on elderly persons with cognitive impairment and dementia: a meta-analysis. Arch Phys Med Rehabil. 2004;85:1694-1704.

4. Wilson BA. Towards a comprehensive model of cognitive rehabilitation. Neuropsychol Rehabil. 2002;12:97-110.

5. Ruthirakuhan M, Luedke AC, Tam A, et al. Use of physical and intellectual activities and socialization in the management of cognitive decline of aging and in dementia: a review. J Aging Res. 2012;2012:384875.

6. US Department of Health and Human Services. Food and Drug Administration. Guidance for Industry, Alzheimer's Disease: Developing Drugs for the Treatment of Early Stage Disease. Silver Spring, MD: US Food and Drug Administration. Available from: http://www.fda.gov/ downloads/drugs/guidancecomplianceregulatoryinformation/guidances/ ucm338287. Accessed April 11, 2015.

7. Weyer G, Erzigkeit H, Kanowski S, et al. Alzheimer's disease assessment scale: reliability and validity in a multicenter clinical trial. Int Psychogeriatr. 1997;9:123-138.
8. Oremus M. Does the evidence say a 4-point change in ADAS-cog score is clinically significant? Alzheimers Dement. 2014;10(3):416-417.

9. Kiresuk J, Smith A, Cardillo JE, eds. Goal Attainment Scaling: Applications, Theory, and Measurement. Hillsdale, NJ: Lawrence Erlbaum Associates; 1994.

10. Rockwood K, Graham JE, Fay S, et al. Goal setting and attainment in Alzheimer's disease patients treated with donepezil. J Neurol Neurosurg Psychiatry. 2002;73:500-507.

11. Rockwood K, Stolee P, Howard K, et al. Use of Goal Attainment Scaling to measure treatment effects in an anti-dementia drug trial. Neuroepidemiology. 1996;15:330-338.

12. Rockwood K, Joyce B, Stolee P. Use of goal attainment scaling in measuring clinically important change in cognitive rehabilitation patients. J Clin Epidemiol. 1997;50(5):581-588.

13. McKhann G, Drachman D, Folstein M, et al. Clinical diagnosis of Alzheimer's disease: report of the NINCDS-ADRDA Work Group under the auspices of Department of Health and Human Services Task Force on Alzheimer's disease. Neurology. 1984;34(7):939-944.

14. Roman GC, Tatemichi TK, Erkinjuntti T, et al. Vascular dementia: diagnostic criteria for research studies: report of the NINDS-AIREN International Workshop. Neurology. 1993;43:250-260.

15. Sahadevan S, Tan NJ, Tan T, et al. Cognitive testing of elderly Chinese people in Singapore: influence of education and age on normative scores. Age Ageing. 1997;26(6):481-486.

16. Mahoney FI, Barthel DW. Functional evaluation: the Barthel Index. Md State Med J. 1965;14:61-65.

17. Lawton MP, Brody EM. Assessment of older people: self-maintaining and instrumental activities of daily living. Gerontologist. 1969;9(3): $179-186$.

18. Kaufer DI, Cummings JL, Ketchel P, et al. Validation of the NPI-Q, a brief clinical form of the Neuropsychiatric Inventory. $J$ Neuropsychiatry Clin Neurosci. 2000;12(2):233-239.

19. Rabin R, de Charro F. EQ-5D: a measure of health status from the EuroQol Group. Ann Med. 2001;33(5):337-343.

20. Zarit SH, Reever KE, Bach-Peterson J. Relatives of the impaired elderly: correlates of feelings of burden. Gerontologist. 1980;20:649-655.

21. Cheah WK, Han HC, Chong MS, et al. Multidimensionality of the Zarit Burden Interview across the severity spectrum of cognitive impairment: an Asian perspective. Int Psychogeriatr. 2012;24(11):1846-1854.

22. Cohen J. Statistical Power Analysis for The Behavioral Sciences. 2nd ed. Howick Place, London: Routledge; 1998.

23. Fritz CO, Morris PE, Richler JJ. Effect size estimates: current use, calculation and interpretation. $J$ Exp Psychol Gen. 2012;141(1):2-18.

24. Holthoff VA, Marschner K, Scharf M, et al. Effects of physical activity training in patients with Alzheimer's dementia: results of a pilot RCT study. PLoS One. 2015;10(4):e0121478.

25. Thuné-Boyle IC, Iliffe S, Cerga-Pashoja A, et al. The effect of exercise on behavioral and psychological symptoms of dementia: towards a research agenda. Int Psychogeriatr. 2012;24(7):1046-1057.

26. Behrer L. Cognitive plasticity in older adults: effects of cognitive training and physical exercise. Ann N Y Acad Sci. 2015;1337:1-6.

27. Clare L, Linden DE, Woods RT, et al. Goal-oriented cognitive rehabilitation for people with early-stage Alzheimer disease: a single-blind randomized controlled trial of clinical efficacy. Am J Geriatr Psychiatry. 2010;18(10):928-939.

28. Arkin S. Language-enriched exercise plus socialization slows cognitive decline in Alzheimer's disease. Am J Alzheimers Dis Other Demen. 2007; 22(1):62-77.

29. Maci T, Pira FL, Quattrocchi G, et al. Physical and cognitive stimulation in Alzheimer disease. The GAIA project: a pilot study. Am J Alzheimers Dis Other Demen. 2012;27(2):107-113.

30. Lau S, Chong MS, Ali N, et al. Caregiver burden: looking beyond the unidimensional total score. Alzheimer Dis Assoc Disord. Epub March 6, 2015.

31. Archbold PG, Stewert BJ, Greenlick MR, et al. Mutuality and preparedness as predictors of caregiver role strain. Res Nurs Health. 1990; 13(6):375-384. 
32. Clare L, Evans S, Parkinson C, et al. Goal-setting in cognitive rehabilitation for people with early-stage Alzheimer's disease. Clin Gerontol. 2011;34(3):220-236.

33. Hartman D. Use of goal-attainment scaling in a dementia special care unit. Am J Alzheimers Dis Other Demen. 1997;12:111-116.

34. Stolee P, Stadnyk K, Myers AM, et al. An individualized approach to outcome measurement in geriatric rehabilitation. J Geront A Biol Sci Med Sci. 1999;54(12):M641-M647.

35. Rockwood K, Stolee P, Howard K, et al. Use of goal attainment scaling to measure treatment effects in an anti-dementia drug trial. Neuroepidemiology. 1996;15:330-338.
36. Bouwens S, van Heugten C, Verhey F. Review of goal attainment scaling as a useful outcome measure in psychogeriatric patients with cognitive disorders. Dement Geriatr Cogn Disord. 2008;26:528-540.

37. Rockwood K, Graham JE, Fay S, et al. Goal setting and attainment in Alzheimer's disease patients treated with donepezil. J Neurol Neurosurg Psychiatry. 2002;73:500-507.

\section{Publish your work in this journal}

Clinical Interventions in Aging is an international, peer-reviewed journal focusing on evidence-based reports on the value or lack thereof of treatments intended to prevent or delay the onset of maladaptive correlates of aging in human beings. This journal is indexed on PubMed Central, MedLine,
CAS, Scopus and the Elsevier Bibliographic databases. The manuscript management system is completely online and includes a very quick and fair peer-review system, which is all easy to use. Visit http://www.dovepress. com/testimonials.php to read real quotes from published authors. 